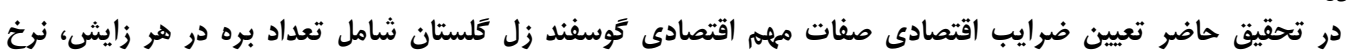

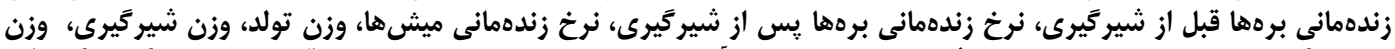

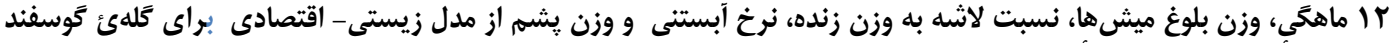

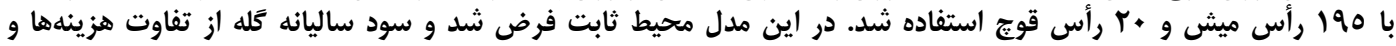

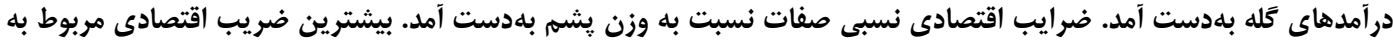

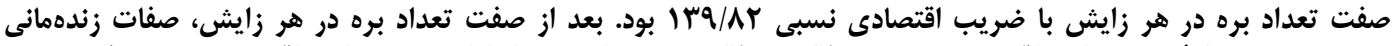

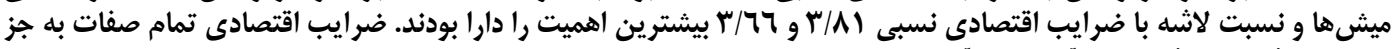

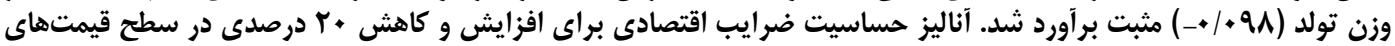

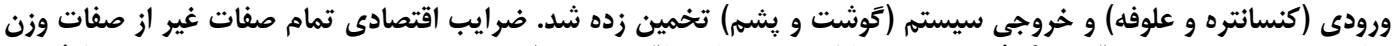

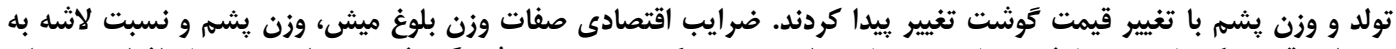

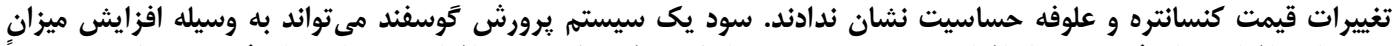

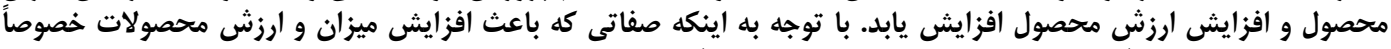

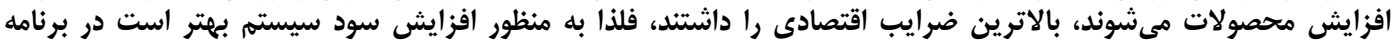
اصلاحنزادى براى اين صفات انتخاب انجام شود.

وازههاى كليدى: كَوسفند، ضرايب اقتصادى، مدل زيستى - اقتصادى

صفت متناسب است (Г). تئورى شاخص انتخاب براى تركيب

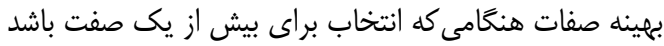

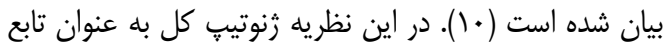

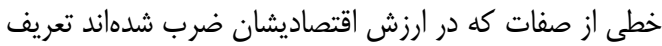

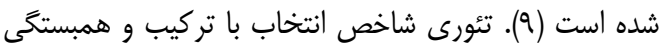

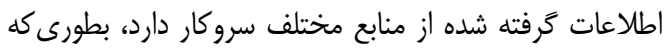

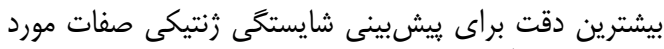

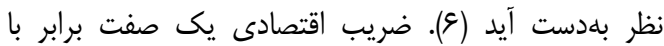

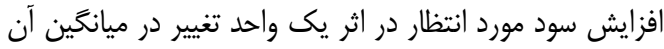

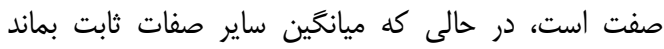

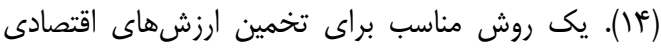

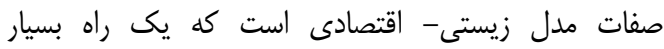

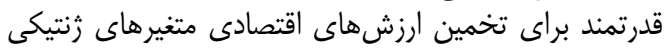

در صفات كوناتون است (1،، (ا، (1).

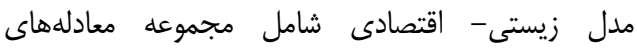
مشخصات بيولوزيكى و وارارمترهاى اقتصادى سيستم است. مزيت عمده اين روش دقت و و قابليت انعطافيذيذيرى و شامل

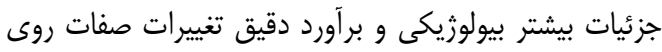

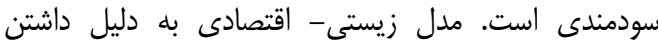

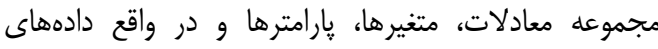
بيشترى را مىتوان در معادلات اين مدل جاى دادئ بنان دابراين،

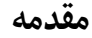

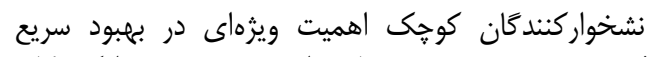

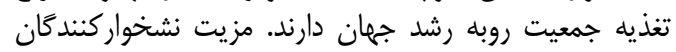

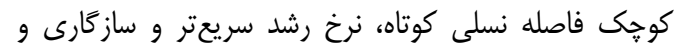

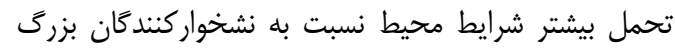

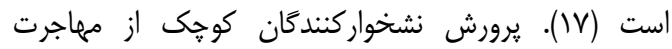

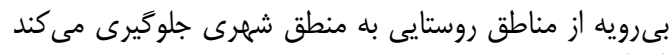

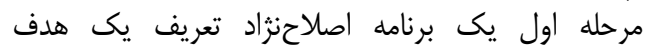

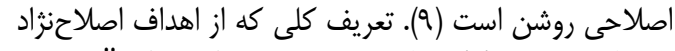

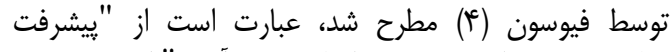

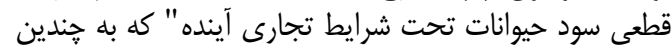

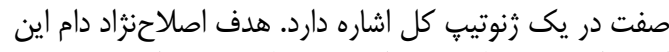

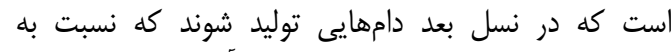

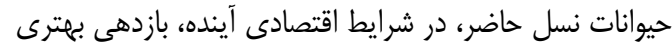

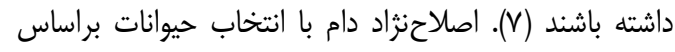

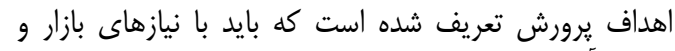

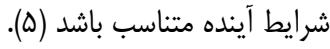

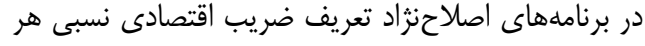

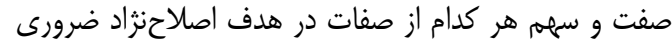

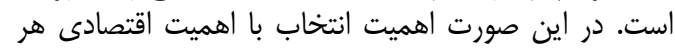


واحد از آن صفت بلهدست مىآيد. در اين مدل محيط ثابت

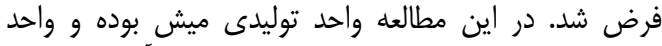

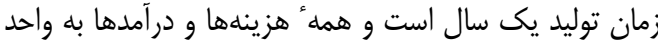

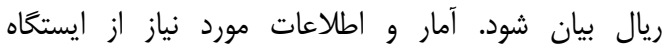

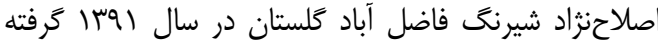

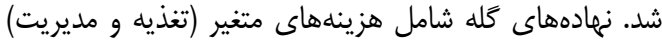

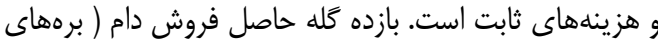

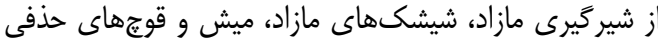

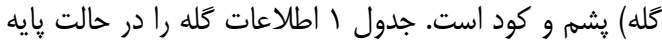

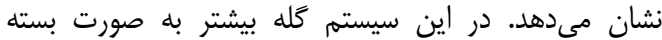

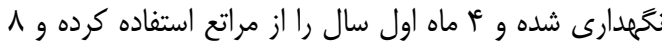
ماه به صورت دستى تغذيه شدهاند.

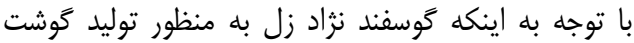

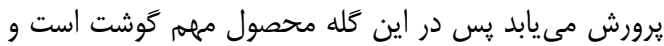

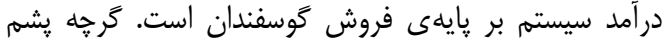

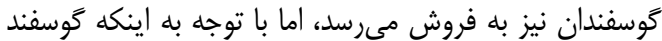

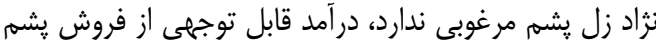

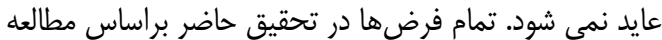
كاسجى و همكاران (Iا (I) است.
اين روش دقيقتر از روشهاى ديخر مثل تابع سود است كه

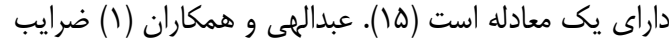

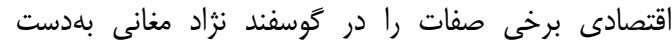

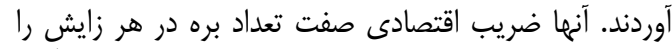
بيشترين ضريب و ضريب اقتصادى وزن تولد را فنفى إنى برآورد

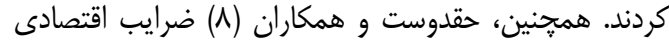

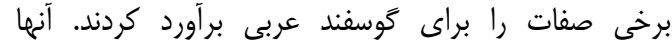

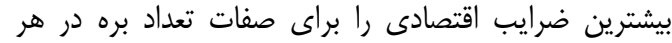

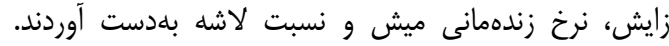

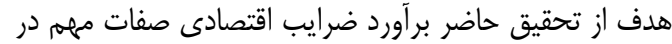

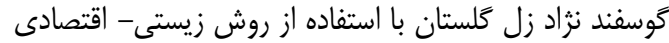

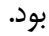

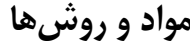 شرح مدل و ساختار كَله}

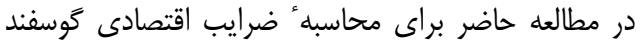
نزاد زل كَلستان، از يكى مدل زيستى - اقتصادى (شبيهسازى ماسيه

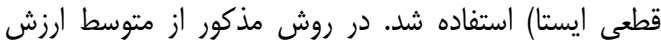

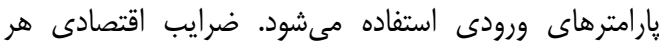
صفت بلوسيله محاسبه سود كله قبل و بعد إد إن تغيير در يكى

Table 1. The structural, production management and nutritional information

جدول ا- اطلاعات ساختارى، توليدى، مديريتى و تغذيهايى

\begin{tabular}{|c|c|c|c|}
\hline 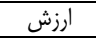 & & 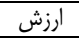 & متغيرها \\
\hline & متغيرهاى مديريتى & & ساختار كله \\
\hline r & سن از شير كيرى (ماه) & 190 & تعداد ميش كله \\
\hline ir & سن اولين جفتخيرى (ماه) & r. & تعداد قوج كله \\
\hline r & تعداد دفعات شמمקينى در سال & $\Lambda \Delta$ & 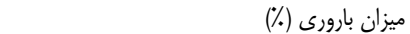 \\
\hline$\Delta$ & تعداد سال هاى" نكَهدارى ميش در كله & Nt & ميزان زايش (٪) \\
\hline$\Delta$ & تعداد سال هاى نكمهدارى قوج در كله & 1 & دفعات برهزايى در سال \\
\hline 1 & نسبت فروش دام ماده در سن r| ماهكى & $1 / 1 r$ & تعداد بره در هر زايش \\
\hline$\cdot / 1$ & نسبت فروش دام نر در سن شير گيرى & ir & دوقلوزايى (\%) \\
\hline \multirow[t]{2}{*}{$\cdot / r$} & نسبت فروش دام نر در سن r| ماهكى & qv & ميزان زندهمانى ميش (\%) \\
\hline & متغيرهاى تنذيهاى & 90 & ميزان زندهمانى قوج (\%) \\
\hline ir. & تعداد روزهاى استفاده از مرتع & 90 & ميزان زندهمانى برهها قبل از شير گيرى (٪) \\
\hline$\cdot$ & تعداد روزهاى استفاده از يِ يسر & 91 & زندهمانى برهها بعد از شير گيرى تا rا ماهگى (\%) \\
\hline rFa & تعداد روزهاى تغذيه دستى & 90 & زندهمانى مادههاى جايكزين (٪) \\
\hline r & متوسط انرزى متابوليسمى علوفه مصرفى (Mcal/DM) & 91 & زندهمانى نرهاى جايخزين (\%) \\
\hline r & متوسط انرزى متابوليسمى مواد دانهاى (Mcal/DM) & & متغيرهاى توليدى \\
\hline eque & متوسط هزينه ( مخاكالرى جيره برهها (Rls./Mcal) & $r / \Lambda$ & وزن تولد (kg) \\
\hline Frr. & متوسط هزينها مكاكالرىجيره شيشكها (Rls./Mcal) & $10 / r^{2}$ & وزن شيركيرى (kg) \\
\hline fiQr & متوسط هزينه امكاكالرىجيره جايكزين (Rls./Mcal) & $\mathrm{r} / \mathrm{V}$ & وزن ri ماهكى دام (kg) \\
\hline זוN & متوسط هزينه ( مكاكالرى جيره ا ميش (Rls./Mcal) & rN/Q & وزن \ا ماهكى ميش (kg) \\
\hline str. & متوسط هزينه ( مكاكالرى جيره اقوج (Rls./Mcal) & $\mu \varphi / 8$ & وزن \ا ماهگى قوج (kg) \\
\hline siti & (Rls./kg DM) قيمت كنسانتره & 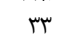 & وزن بلوغ ميش (kg) \\
\hline \multirow[t]{2}{*}{ mas } & (Rls./kg DM) قيمت علوفه & rr & وزن بلوغ قوج (kg) \\
\hline & 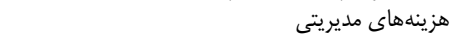 & is & بازده لاشه (\%) \\
\hline r.... & هزينه دارو و درمان (Rls./head/year) & 1/ब & 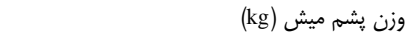 \\
\hline r.... & هزينه واكسيناسيون (Rls./head/year) & r & وزن پشم قوج (kg) \\
\hline $195 .$. & هزينه كنترل بهداشتى (Rls./head/year) & $1 / r$ & وزن קشم شيشك/ دام ماده جايكزين (kg) \\
\hline rASTI. & هزينه كاركرى (Rls./100head/month) & $1 / \Delta$ & وزن يشم نر جايگزين (kg) \\
\hline \multirow[t]{3}{*}{ t.DIT } & هزينه حمل خوراى (Rls./head/year) & 10 . & تعداد روزهاى آبستنى \\
\hline & &.$/ 49$ & ميزان شير ميش دارآى بره تك قلو (kg/day) \\
\hline & &.$/ 4 F$ & ميزان شير ميش داراى بره دوقلو (kg/day) \\
\hline
\end{tabular}




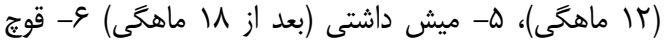

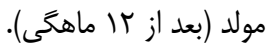

معادلات سود (ردل مود

كل سود ساليانه' سيستم توسط معادله زير بلهدست آمد

$\mathrm{Tf}=[\mathrm{Ne} \times(\mathrm{Re}-\mathrm{Ce})]-\mathrm{C}_{\mathrm{FCF}}$

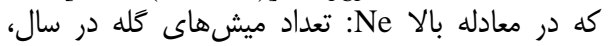

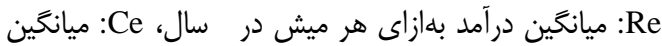

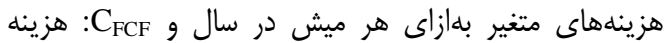
ثابت كله است.

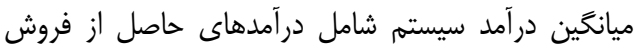

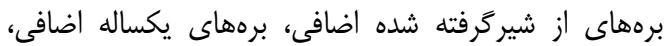

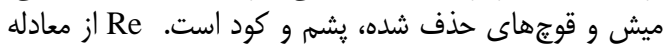
زير بهدست مى آيد (Iا):

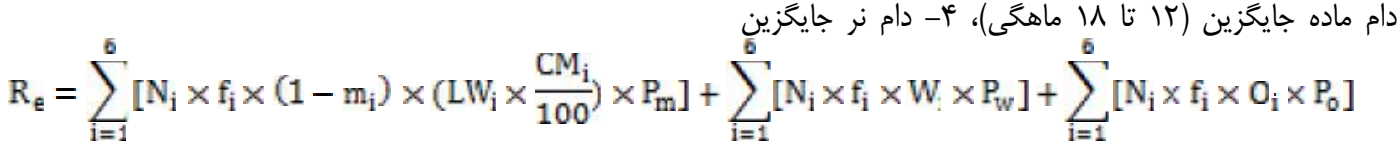

كه در معادله بالا CC: هزينه تغذيه (علوفه و كنسانتره)،

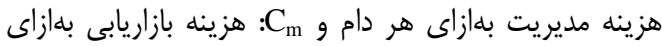

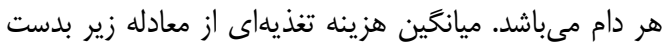
$C_{f}=\sum_{\mathrm{i}=1}^{6}\left[N_{\mathrm{i}} \times R F_{\mathrm{i}} \times L_{\mathrm{i}} \times P_{r f}\right]$

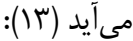

: احتياجات ماده خشك علوفه خشبى و كنسانتره براى

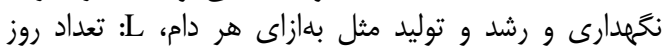

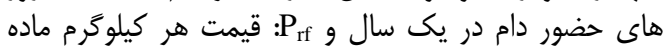
خشى كنسانتره و علوفه خشبى ديى مى دباشد.

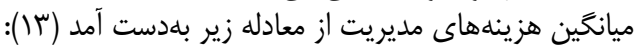

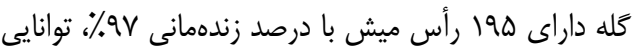

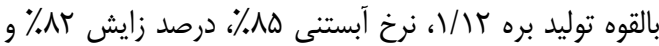

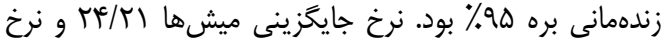
جايخزينى قوجها بلهورت يك بار زايش در سال است.

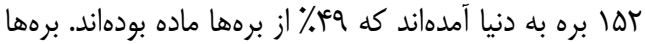

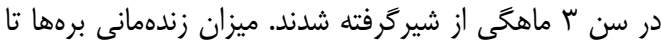

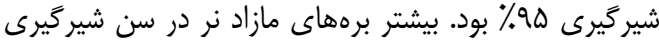

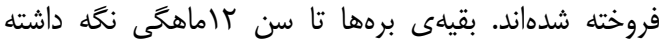

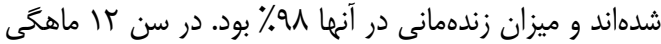

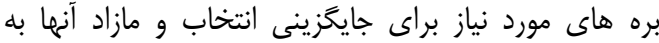

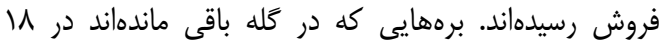
ماهخى جايخزين ميش هاى حذفيد نده شدهاند.

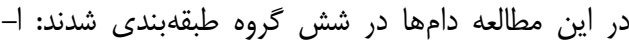

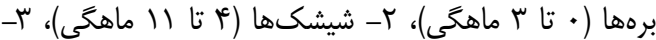
در معادله بالا i : طبقهبندى دامها در كله، N:

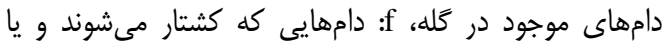
بخشى از دامها كه به منظور توليد كود كشاورزى، كود دود توليد

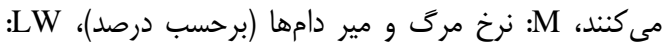
وزن زنده دامهاى كشتارى (كيلوگرم)،

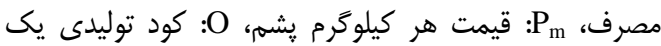

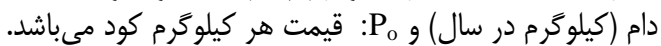
هزينههاى متغير (C)

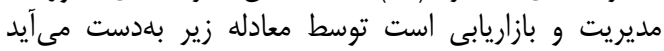
$C_{e}=\sum_{\mathrm{i}=1}^{\sigma} N_{\mathrm{i}} \times\left[\mathrm{C}_{\mathrm{f}_{\mathrm{i}}}+\mathrm{C}_{\mathrm{h}_{\mathrm{i}}}+\mathrm{C}_{\mathrm{m}_{\mathrm{i}}}\right]$

$\left.\mathrm{C}_{\mathrm{h}}=\sum_{\mathrm{i}=1}^{\mathrm{\sigma}}\left[\mathrm{N}_{\mathrm{i}} \times\left\{\mathrm{P}_{\mathrm{bi}}+\mathrm{C}_{w_{\mathrm{c}_{\mathrm{i}}}}+\left(\mathrm{N}_{\mathrm{d}: y_{\mathrm{i}}} \times \mathrm{C}_{\mathrm{d}_{\mathrm{i}}}\right)+\mathrm{C}_{\mathrm{vi}}\right)+\left(\mathrm{D}_{\mathrm{ml}_{\mathrm{i}}} \times \mathrm{L}_{\mathrm{i}} \times \mathrm{P}_{\mathrm{ml}}\right]\right\}\right]$

$\mathrm{C}_{\mathrm{m}}=\sum_{\mathrm{i}=\mathrm{L}}^{\sigma}\left[\mathrm{N}_{\mathrm{i}} \times \mathrm{f}_{\mathrm{i}} \times\left(\mathrm{C}_{\mathrm{t}_{\mathrm{i}}}+\mathrm{C}_{\mathrm{l}_{\mathrm{i}}}\right)\right]$

نقل دام زنده بداد دامهايى كه فروخته مىشوند، C:

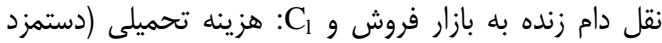

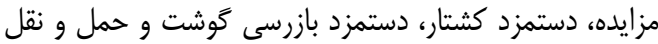

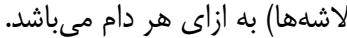

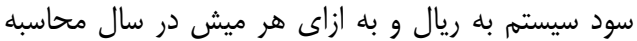
شده است. ضريب اقتصادى هر صفت از تفاوت سود سيستم

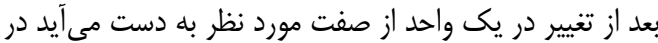

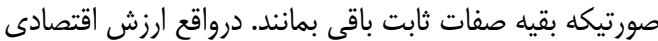

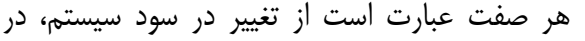

$(\mathrm{EV})$
در معادله بالا Pال رأس دام، C C

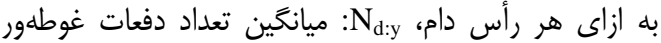
كردن در حمام هاى ضد كنه در هر هر رأس دام،

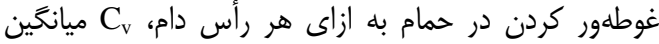

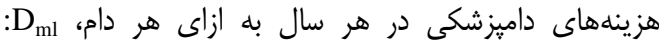

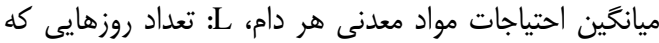

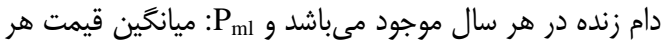
كيلو مرم مواد معدنى است.

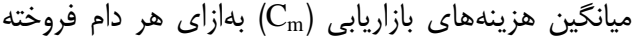
شده توسط معادله زير برآورد مى شود (سار): 


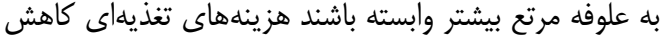

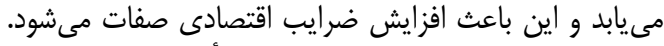

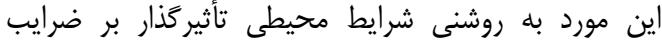

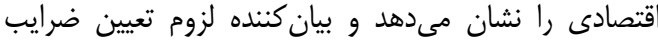

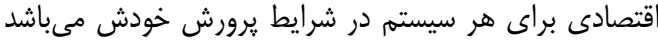

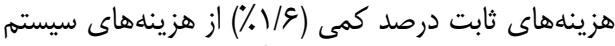

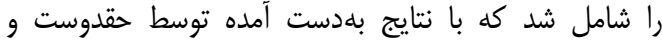

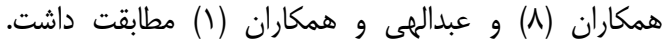

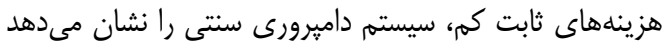

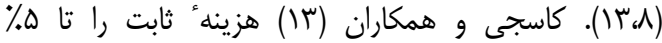

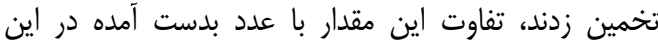

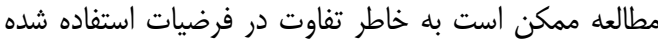

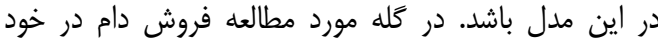

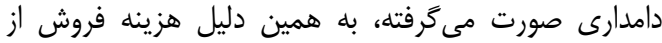

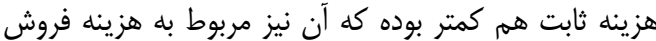

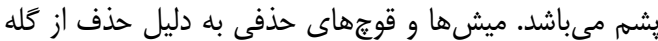

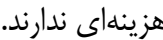

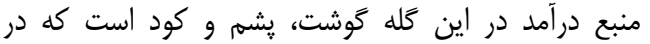

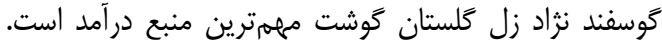

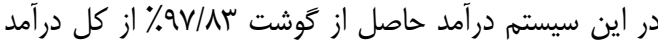

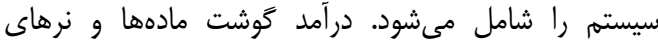

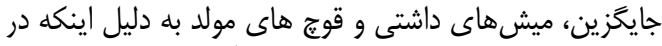

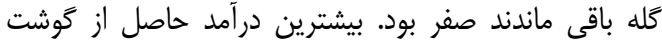

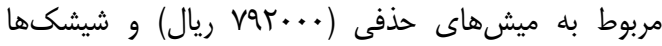

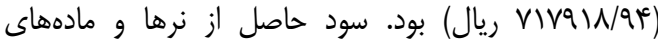
جايخزين، ميش داشتى و قوج مولد به دليل ماندن ساصن در كله

$$
\text { منفى شد. }
$$

نتيجه يك واحد تغيير در صفت مورد نظر در صورتيكه ساير

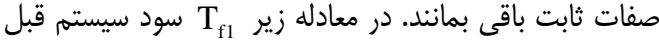

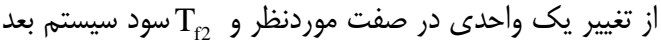
از تغيير يك واحدى در صفت مورد نظع درى مورد است. ضريب اقتصادى هر صفت در واقع از تفاوت T2 $E V=T_{f 2}-T_{f 1}$

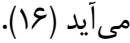

\section{نتايج و بحث موقعيت سود يايه}

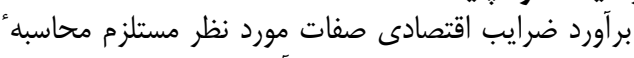

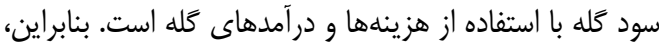

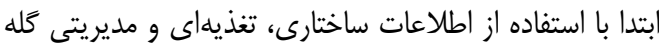

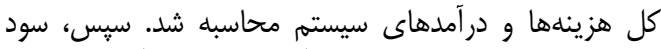

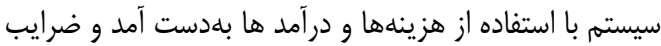

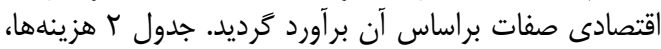

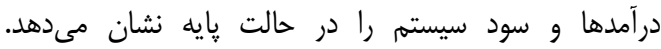

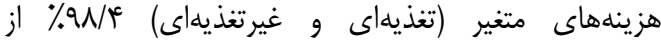

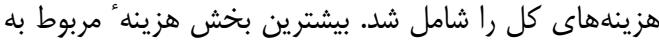

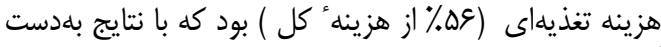

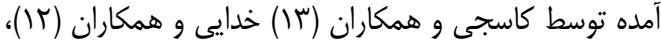

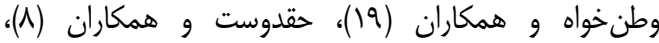

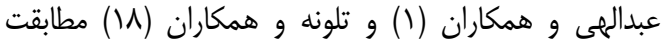

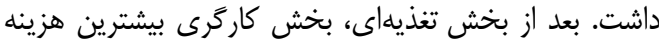

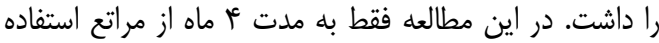

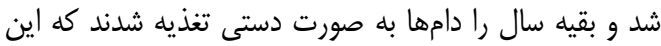

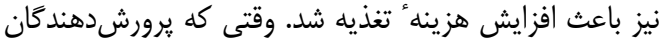

Table 2. Costs, revenues and profits for the Zel sheep of Golestan

جدول r- هزينهها، درآمدها و سود كَله كَوسفند نزاد زل كَلستان

\begin{tabular}{|c|c|c|c|c|c|c|c|c|c|}
\hline & حذفّى & حذفى ميش & قوج & ميش داشتى & نر جايكزين & ماده جايخزين & شيشكها & برهها & \\
\hline & $\cdot / \cdot r \cdot \Delta \mid$ & $\cdot / T$ & $. / 1 . T \Delta F$ & 1 & ./.rEIEATIT & . /TETI.QTEK & $\cdot / F \Delta>99>9 \Delta$ & $\cdot / V^{*} \mid \overline{1} \cdot 1$ & نسبت كروه به \\
\hline كل & & & & & & & & & هزينهها \\
\hline$q \cdot V \cdot q \mu$ & ...... & $\ldots .$. & $\Delta 9991 / 9$ & QTTIN/ATq & $|r \Delta| F / . r q T q$ & $1 . \wedge F \Delta V / \Delta \backslash \Delta S$ & 10DTQG/\& & $r \wedge g V / / r q$ & تغذيهاى \\
\hline sNDITL/V & $19 \% / 1 . \mu$ & $19 .$. & FIDII/. & 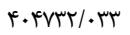 & $N r \cdot 1 / r \cdot r \cdot v 1$ & VDMAV/AqIIV & 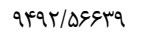 & $\Delta \Lambda 11 \cdot / \Lambda \mu$ & غيرتنذيهاى \\
\hline$r \Delta V \cdot r / V$ & $\ldots . .$. & $\ldots . .$. & lQTYK/VE & $1 F q S \% / V \Delta D Q$ & $r q T / g$ TVTAlq & $r V I V / N \cdot r \cdot r r$ & MFIQ/I9DFFV & $r W Y \in / \mu 1$ & ثابت \\
\hline 19189.1 & $\mid 9 \% / 1 . \mu$ & $19 .$. & 1. rquV & QFIAVV/GTV & r)..N/AVaVe & $M V \cdot G K / \Delta \cdot M$ & TQMGAT/TMT & $1.9 \Delta \Delta \sigma / 4$ & كل \\
\hline$r \mid \cdot \lambda \kappa .$. & $91 \cdot V \varepsilon / 9$ & Var... & ..... & ...... & ...... & ...... & VIV9IN/9FV & $\Delta \cdot V F \cdot F / \Delta$ & كوشت \\
\hline TQTFY/T & $F 1 . / T \Delta S$ & $r \ldots$ & $r \cdot \Delta l / T \Lambda$ & $10 \ldots$ & rqT/\&AYGVAr & 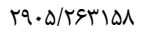 & DFAT/QGMFET & ...... & بشم \\
\hline $\mid V \Psi q \cdot / V$ & $\ldots . .$. & ..... & 1.19/9 & ITFFN/TH.D & 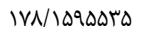 & IDFT/GGMMIF & $1 F \cdot V / F A T \Delta q D$ & $994 / 1190$ & كود \\
\hline$r \mid \Delta Q \ldots r$ & $91 F A V / T$ & v9a $\ldots$ & MIFI/TI & TYFEN/THOQ D & $\Delta V \cdot / 9949 T I V$ & FFeV/quIqVI & $V T F \Lambda 1 . / r q T V$ & $0 \cdot 1 \cdot 9 \mathrm{~N} / \mathrm{V}$ & كل \\
\hline$\Delta T V I \cdot r$ & 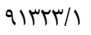 & varf.. & $-994 \% 8$ & -q)ferq/r & $-r \cdot F r N / T \Delta Q 1$ & $-|A Y G| F / \Delta V G$ & FVIles/IVIV & एQADFT/T & سود \\
\hline
\end{tabular}


(درآمد حاصل از گوشت) شد در صورتيكه هزينهها تغييرى

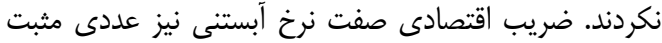

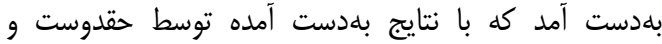

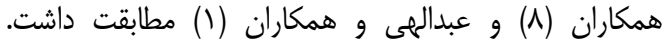

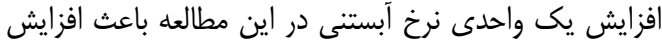

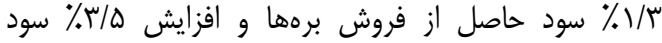

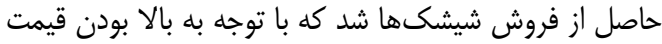

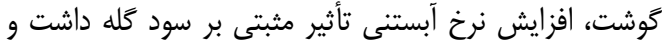

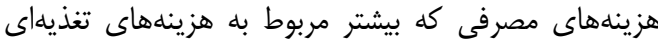

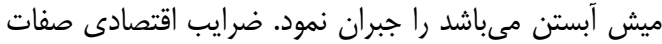

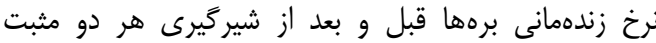

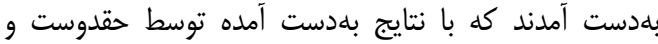

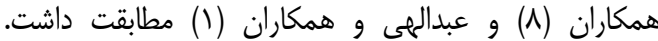

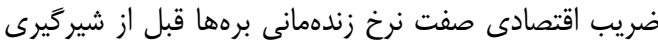

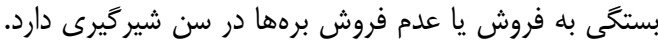

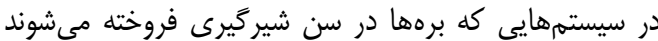

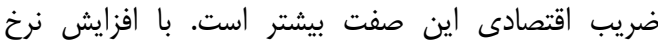
زندهانى برهها، تعداد برههاى قابل فروش بيشت بيشر شد، در

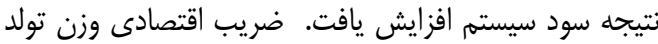

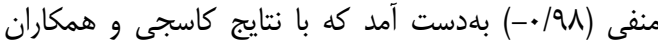

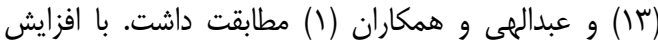

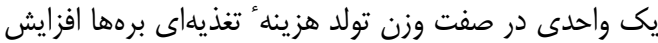

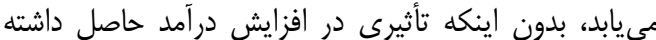

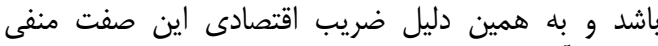

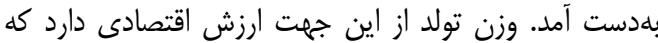

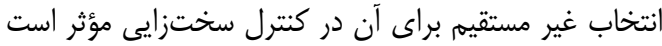

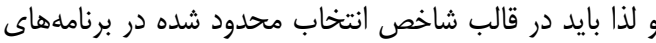

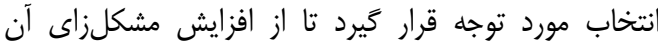

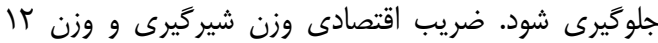

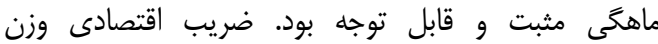

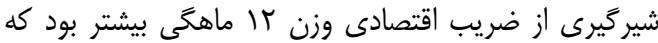

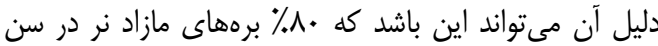

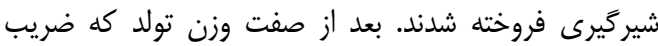

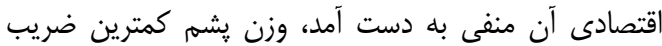

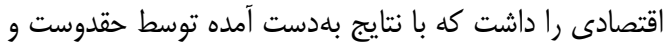

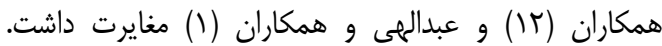

ضر ايب اقتصادى

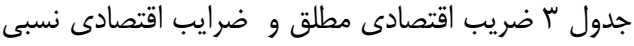

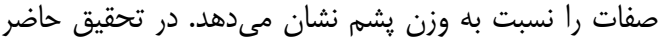

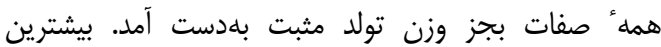

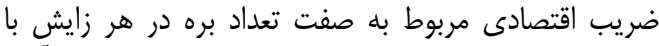

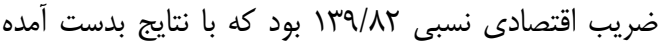

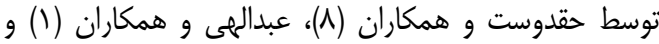

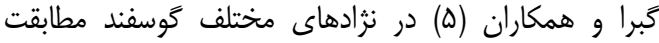

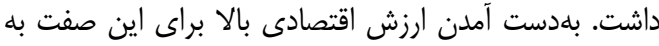

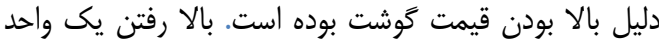

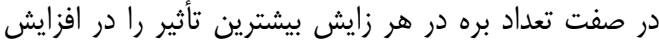

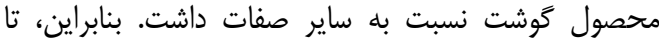

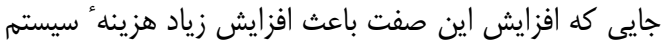

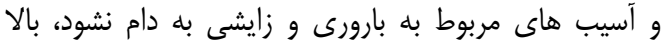
بردن اين صفت در برنامههاى اصلاحى بسيار مفيد و سودمند

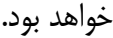

بعد از صفت تعداد بره در هر زايش، صفات زندات زندهانى ميشها و نسبت لاشه به وزن زنده بيشترين ضريب اقته

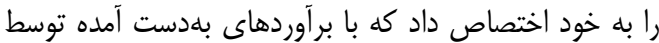

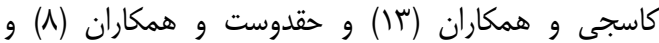

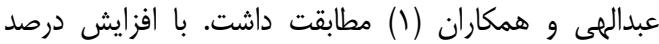

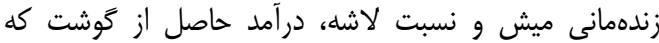

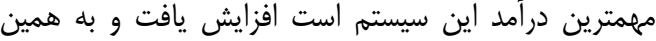

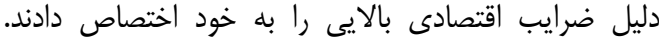

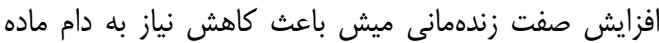

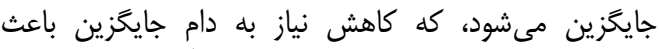

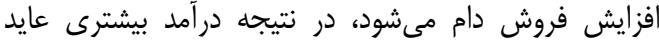

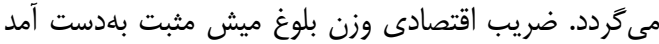

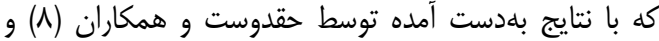

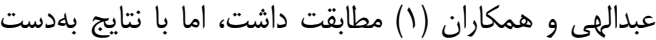

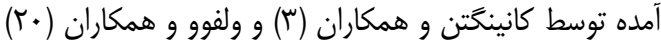

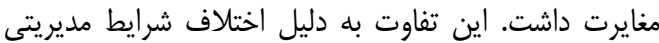

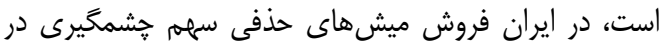

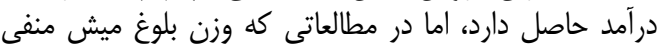

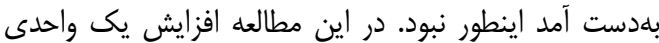
وزن بلوغ ميش باعث افزايش سود حاصل از ميش ايشهاى ايش حذفى

Table 3. Absolute and relative economic values of traits

جدول ץ- ضرايب اقتصادى مطلق و نسبى صفات

\begin{tabular}{|c|c|c|}
\hline ضريب اقتصادى نسبى & ضريب اقتصادى مطلق (ريال) & صفات \\
\hline $1 r q / A r$ & $19 V V 9 \Delta \mathrm{V}$ & تعداد بره در هر زايش \\
\hline $1 / 99$ & $r \cdot r \Lambda r^{2}$ & نرخ آبستنى (٪) \\
\hline $1 / 84$ & IQYAV & نرخ زندهانى قبل از شيرگيرى (٪) \\
\hline $1 / r \Delta$ & DQ.r. & نرخ زندهانى بعد از شيرگيرى (\%) \\
\hline$r / \Lambda)$ & FANTK & زندهمانى ميش (\%) \\
\hline.$- / 94$ & $-11 V \Delta$ & وزن تولد (kg) \\
\hline$r / 4 Q$ & rवि९s & وزن شيرگيرى (kg) \\
\hline $1 / \Delta \Lambda$ & 19.4. & وزن يكسالكى (kg) \\
\hline$r$ & $r \leftarrow \ldots$ & وزن بلوغ ميش (kg) \\
\hline 1 & $1 \% \ldots$ & وزن يشم ساليانه ميش (kg) \\
\hline$r / 99$ & מזם & بازده لاشه (\%) ب \\
\hline
\end{tabular}




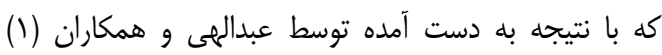

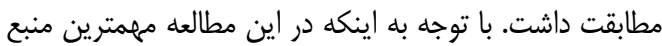

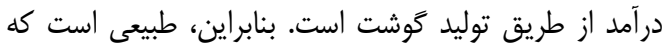

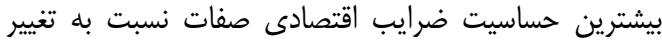

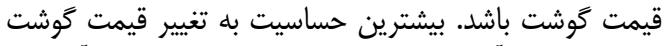

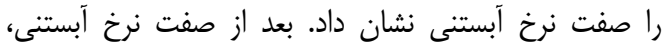

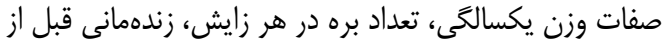

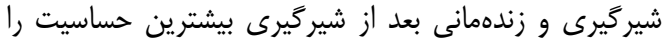

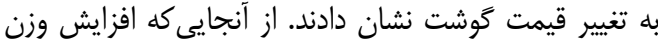

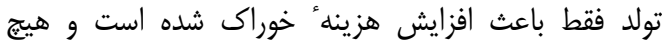

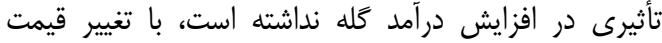

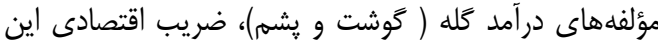

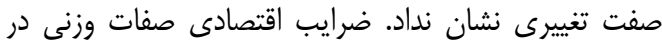

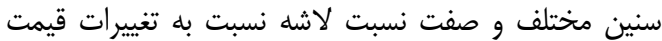
شֶم حساسيت نشان ندادند.

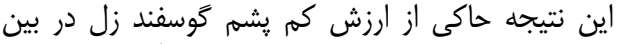

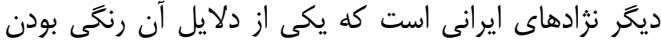

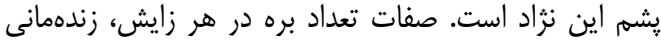

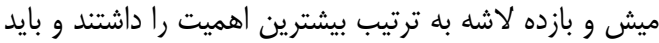

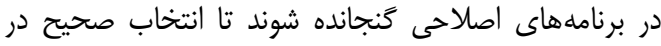

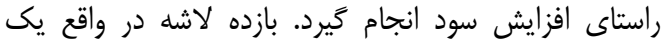

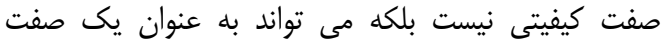

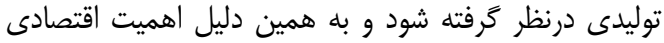
بالايى داشته است. آناليز حساسيت جدول أ حساسيت ضرايب اقتصادى را نسبت به به تغييرات

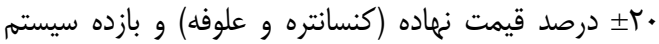

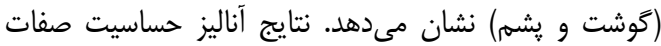

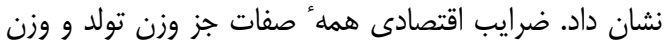

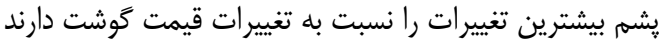

جدول أ- آناليز حساسيت ارزشهاى اقتصادى صفات نسبت به تغيير در سطح قيمتهاى نهاده و محصول Table 4. The sensitivity analysis economic value than traits with changes in levels of inputs and outputs prices

\begin{tabular}{|c|c|c|c|c|c|c|c|c|c|c|c|c|c|}
\hline \multicolumn{11}{|c|}{ صفات } & \multirow[t]{2}{*}{ سود } & \multirow[t]{2}{*}{ قيمت (\%) } & \multirow[t]{2}{*}{ محصول } \\
\hline LS & CR & PRWS & PWS & ES & BW & WLW & $12 \mathrm{mLW}$ & ELW & WW & DP & & & \\
\hline$r \cdot V \backslash \Lambda \cdot \Lambda$ & TQDVF & Tefer & $\mid \wedge F \wedge \Delta$ & DTAT. & $-11 V \Delta$ & reIVTr & TrQV. & $T M .$. & $1 r \ldots$ & DTVI. & १QAVA & $+r$. & كَوشت \\
\hline $\mid$ IFAFI.G & 10190 & DQtef & $110 \Delta \Delta$ & rVAIr & $-11 V \Delta$ & rrqQ & $\mid F \Delta \|$ & 19\%.. & $r \ldots$ & rolf. & INAFTH & r. & \\
\hline $19 \vee 1191$ & r. एव, & 19791 & $10 \cdot \%$. & forge & $-11 V \Delta$ & rqDS9 & 19.4. & $r f \ldots$ & lef.. & FTaTD & DFYQDT & $+r$. & קֶشم \\
\hline $19 W \cdot \pi r$ & $r . r V I$ & lawe & $10 \cdot \cdot 1$ & $F \Delta \Lambda F$ & $-11 V_{\Delta}$ & TQD\&D & 19.4. & $r F \ldots$ & १९.. & kTato & $\Delta M \mid T \Delta \Delta$ & $-r$. & \\
\hline $199 \cdot 1 \mid \mathrm{V}$ & 194TV & 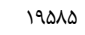 & $|F M|$ & $4 \Delta 9.1$ & זוזו- & TITIT & WVYG & $r f \ldots$ & $r \ldots$ & Frats & $F \Delta F \cdot I T$ & $+r$. & كنسانتره \\
\hline 1990.98 & r.9Fr & 19919 & $|\Delta| \Delta A$ & forres & $-1 \cdot r v$ & г१९ร. & 19494 & $r f \ldots$ & $1 r \ldots$ & FraTs & 95.194 & $-r$. & \\
\hline 199. & r.1FT & 19011 & | & D950 & - ITVG & גוזוא & WFF" & $r+\ldots$ & $r \ldots$ & reata & FellTs & $+r$. & علوفه \\
\hline $189 \Delta F$. & T.9TA & 19994 & lolVV & tor.t. & $-1 \cdot v^{c}$ & ז१९ाए & 194FD & $r F \ldots$ & $\| r \ldots$ & reata & $9 \pi+\Lambda 1$ & $-r$. & \\
\hline & & & & $\checkmark$ & & & $=$ & شه & $\begin{array}{l}\text { :PRWS } \\
: W L W\end{array}$ & & $\begin{array}{l}\text { CR } \\
\text { :BW } \\
\text { : }: \text { :WW }\end{array}$ & ميش & $\begin{array}{l}\text { ES } \\
\text { ELS } \\
\text { ELد : ELW }\end{array}$ \\
\hline
\end{tabular}

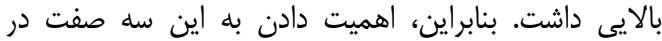

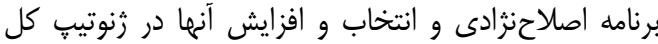

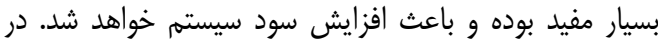

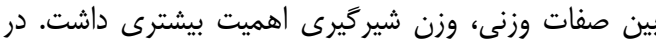

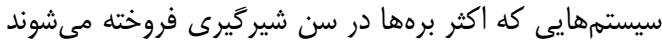

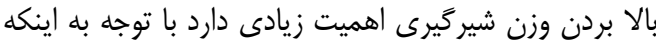

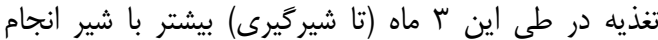
مى

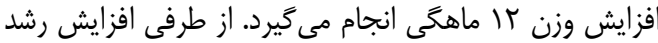

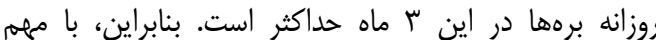

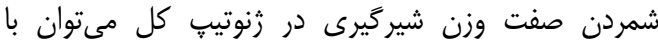

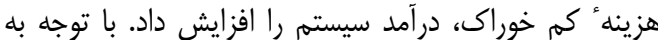
منفى بودن وزن تولد افزايش اين صفت برائ باعث افت افت سود

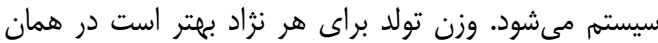

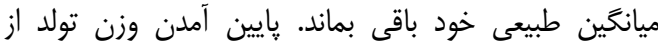

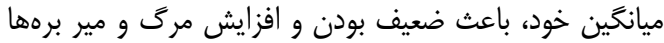

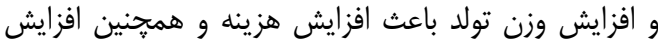

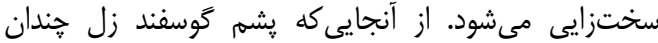

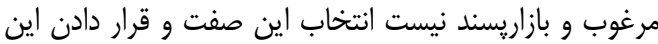
صفت در زنوتيڤ كل تأثير جندانى در سود سيستم ندارد.

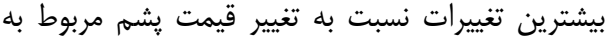

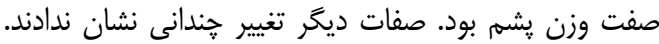

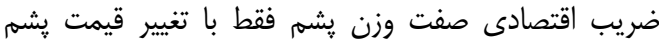

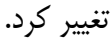

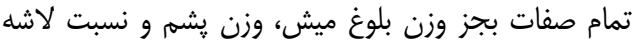

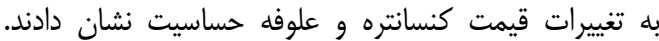
بيشترين تغييرات نسبت به قيمت كنسانتره و علوفه مربوط به به

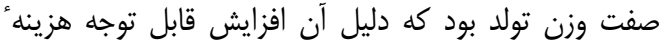

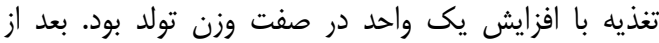

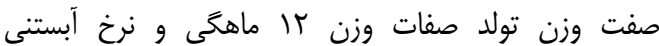

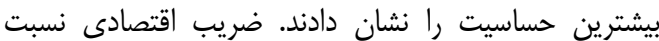
لاشه فقط با تغيير قيمت گوشت تغيت الغيير كرد. آناليز حساسيت

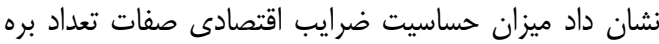

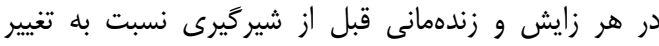
قيمت همه 'مؤلفهها (كوشت، يشم، كنسانتره و علوفه) يكسان

صفات تعداد بره در هر زايش و زندهمانى ميش بيشترين

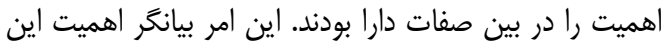

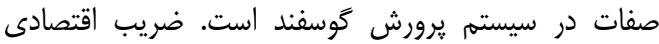

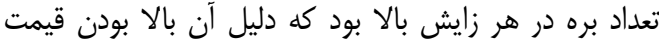

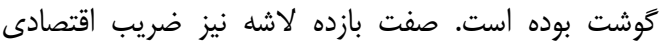




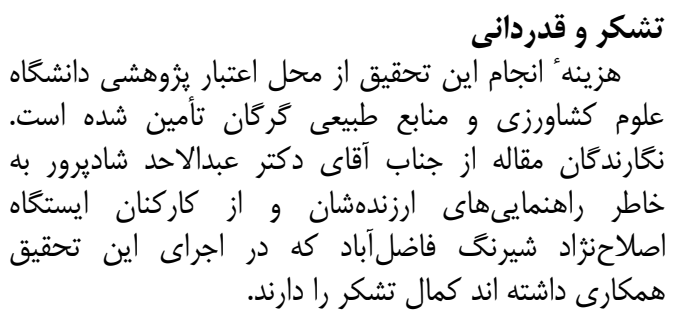

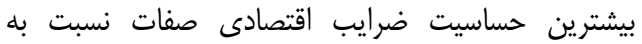

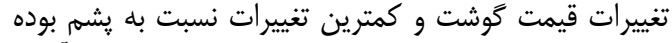

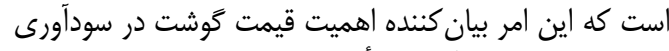

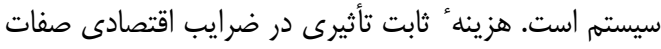

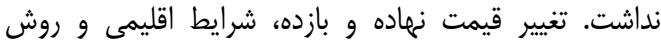

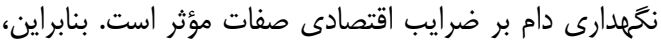

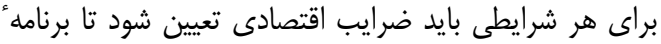

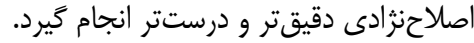

1. Abdolahy, H., S. Hasani, S. Zerehdaran, A.A. Shadparva and B. Mahmoudi. 2012. Determination of economic values for some important traits in Moghani sheep. Small Ruminant Research, 105: 161169.

2. Amer, P.R., G. Simm, M.G. Keane, M.G. Diskin and B.W. Wickham. 2001. Breeding objectives for beef cattle in Ireland. Livestock Production Science, 67: 223-239.

3. Conington, J., S.C. Bishop, A. Waterlouse and G. Simm. 2004. A bio-economic approach to derive economic values for pasture-based sheep genetic improvement programs. Journal of Animal Science, 82: $1290-1304$.

4. Fewson, D. 1993. Definition of the breeding objective. Design of livestock breeding programmes. Animal Breeding and Genetics Unit, University of New England, Armidale, NSW, Australia.

5. Gebra, K.T., B. Fuerst-Waltlb, M. Wurzingerb, J. Philipssonc, G. Dugumad, T. Mirkenae, A. Hailef and J. Solknerb. 2012. Estimates of economic values for important indigenous Ethiopian sheep breeds. Small Ruminant Research, 105: 154-160.

6. Gibson, J.P. 1995. An introduction the design and economic of animal breeding strategies. University of Guelph, Canada.

7. Groen, A.F., T. Steine, J.J. Colleau, J. Pederson, J. Pribyl and N. Reinsch. 1997. Economic value in dairy cattle breeding with special reference to functional traits. Report of EAAP Working Group. Livestock production science, 49: 1-21.

8. Haghdoost, A., A.A. Shadparvar, M.T. Beigi Nasiri and J. Fayazi. 2008. Estimates of economic values for traits of Arabic sheep in village system. Small Ruminant Research, 80: 91-94 (In Persian).

9. Hazel, L.N. 1943. The genetic basis for constructing selection indexes. Genetics, 28: 476-490.

10. Hazel, L.N. and J.L. Lush. 1943. The efficiency of three methods of selection. Journal of Heredity, 33: 393-399

11. Jones, H.E., P.R. Amer, R.M. Lewis and G.S. Emmans. 2004. Economic values for changes in carcass lean and fat weight at a fixed age for terminal sire breeds of sheep in the UK. Livestock Production Science, 89: 1-17.

12. Khodaee, M. 2005. Determination of breeding objectives of Guilanian sheep by estimation of economic coefficients of production traits. M.Sc. Thesis, Guilan University, Guilan, Iran (In Persian).

13. Kosgey, I.S., J.A.M. Van Arendonk and R.L. Baker. 2003. Economic values for traits of meat sheep in medium to high production potential areas of the tropics. Small Ruminant Research, 50: 187-202.

14. Kosgey, I.S., J.A.M. Van Arendonk and R.L. Baker. 2004. Economic values for traits in breeding objectives for sheep in the tropics: impact of tangible and intangible benefits. Livestock production Science, 88: 143-160.

15. Krupova, Z., M. Oravcora, E. Krupa and D. Peskovicova. 2008. Methods for calculating economic weight of important traits in sheep. Slovak Journal Animal Science, 41: 24-29.

16. Ponzoni, R.W. 1992. Genetic improvement of hair sheep in the tropics. FAO Animal Production and Health Papers 101, Rome, Italy 168 pp.

17. Tibbo, M. 2006. Productivity and health of indigenous sheep breeds and crossbreds in the central Ethiopian highlands. Ph.D. Dissertation, Department of Animal Breeding and Genetics, Faculty of Veterinary Medicine and Animal Sciences. Swedish University of Agricultural Sciences (SLU), Uppsala, Sweden. http:// pub. epsilon. slu. se/1142/1/ Markos_ Tibbo_corrected. Pdf Accessed on December 5, 2011.

18. Tolonea, M., V. Riggioa, D.O. Maizonc and B. Portolanoa. 2011. Economic values for production and functional traits in Valle del Belice dairy sheep using profit functions. Small Ruminant Research, 97: 41-47.

19. Vatankhah, M. 2005. Defining a proper breeding scheme for Lori-Bakhtiari sheep in village system Ph.D. Thesis, Tehran University, Tehran, Iran (In Persian).

20. Wolfová, M., J. Wolf and M. Margetín. 2009. Estimation of economic values for traits of dairy sheep: II. Model application to a production system with one lambing per year. Journal of Dairy Science, 92: 2195-2203. 


\title{
Determination of Economic Values for Some Important Traits in Zel Sheep of Golestan
}

\author{
Behnaz Zarrinkamar ${ }^{1}$, Saeed Hassani ${ }^{2}$, Saeed Zerehdaran ${ }^{3}$, Hossein Abdolahy ${ }^{4}$ and \\ Rahmat Samiei $^{5}$ \\ 1- Graduate M.Sc. Student, Gorgan University of Agricultural Sciences and Natural Resources \\ (Corresponding author: zarrinkamar.b@gmail.com) \\ 2- Associate Professor, Gorgan University of Agricultural Sciences and Natural Resources \\ 3- Associate Professor, Ferdowsi University of Mashad \\ 4- Ph.D. Student Zabol University \\ 5- Deputy of Livestock Division, Golestan Jahad-Agriculture Organization \\ Received: Thursday, July 24, $2014 \quad$ Accepted: June 7, 2015
}

\begin{abstract}
In this study a bio-economic model was used for a flock with 195 breeding ewes and 20 rams to determine economic values of important traits in Zel sheep of Golestan including litter size, pre-weaning survival, post-weaning survival, ewe survival, birth weight, weaning weight, yearling weight, ewe mature weight, dressing percentage, conception rate and wool weight. In this model, fixed environment was assumed and herd profit was estimated from difference between costs and revenues. Relative economic values of the traits were estimated based on wool weight. The highest economic value was found for litter size with relative economic value of 139.82. After litter size, the most important relative economic values were 3.81 and 3.66 for ewe survival and dressing percentage, respectively. Economic values of all traits were positive except for birth weight (-0.98). Sensitivity analysis of economic values were carried out for $20 \%$ increase and decrease in price levels of input (concentrate, forage) and output (meat, wool) system. Economic values of all traits except for birth weight and wool weight were changed by changing the price of meat. The economic values of ewe mature weight, dressing percentage and wool weight did not change with change in price of concentrate and forage. Sheep farming system can increase in profits by increasing in the amount and value of product. As the highest economic values were found for amount and value of the products especially amount of products, in order to increase system profit it is better to select for these traits in a breeding program.
\end{abstract}

Keywords: Bio-economic model, Economic values, Sheep 